\title{
Preparation of Nanoiron by Water-in-Oil (W/O) Microemulsion for Reduction of Nitrate in Groundwater
}

\author{
Tielong LI ${ }^{12}$, Shujing $\mathrm{LI}^{1}$, Shuaima WANG ${ }^{1}$, Yi AN ${ }^{1}$, Zhaohui $\mathrm{JIN}^{\mathbf{1}}$ \\ ${ }^{1}$ College of Environmental Science and Engineering, Nankai University, Tianjin, China \\ ${ }^{2}$ Tianjin Key Laboratory of Environmental Remediation and Pollution Control/Ministry of Education Key Laboratory of \\ Environmental Processes and Criteria, Nankai University, Tianjin, China \\ E-mail: litielong@nankai.edu.cn \\ Received December 8, 2008; revised February 2, 2009; accepted February 12, 2009
}

\begin{abstract}
Nanometer-sized iron particles with monodispersity and narrow size distribution were synthesized by modified microemulsion system using environment-friendly non-ionic surfactants Span 80 and Tween 60 as mixed surfactants. The synthesized iron nanoparticles were characterized by using powder X-ray diffraction (XRD) and transmission electron microscopy (TEM). The results show that the synthesized particles were mainly composed of $\alpha-\mathrm{Fe}$ with an average diameter of 80-90 nm. The chemical activity of the obtained iron nanoparticles was evaluated by the denitrification of nitrate in water. On neutral condition, complete denitrification of nitrate was achieved by freshly synthesized nanoiron within $30 \mathrm{~min}$. Ammonia was the main product, with good material balance at the end of the reaction. Two possible reaction pathways for nitrate reduction by nanoiron have been proposed in this study.
\end{abstract}

Keywords: Iron Nanoparticles, Microemulsion, Reduction, Nitrate

\section{Introduction}

Metallic nanoparticles play important roles in many different areas by virtue of their unique microstructure (small size effect, quantum effect, and surface effect). The physical and chemical properties of the nanoparticles are different from those of bulk metal due to their extremely small sizes and large specific surface areas. They have been widely used in the areas of catalysis, luminous materials, high quality magnetic materials, electronics, biomedical engineering and environmental remediation [1-4]. Recently, the use of nanoscale materials for in-situ remediation of non-aqueous phase liquids (NAPLs) [5-7] and heavy metals (e.g. As, $\mathrm{Cr}$, and $\mathrm{Pb}$ ) $[8,9]$ in groundwater has been deeply and extensively studied, and much progress has been achieved.

Water-in-oil (W/O) microemulsions or reverse micelles, which are also called intelligent microreactors $[10,11]$, are of particular interest in fabricating nanoparticles. The W/O microemulsions consist of nanosized water droplets that are dispersed in a continuous oil medium and stabilized by surfactant mole- cules accumulated at the oil/water interface. A variety of reactants can be introduced into nanosized water droplets for reactions confined within the microreactors, leading to materials with uniform size. The highly dispersed water pools are ideal nanostructured reaction media for producing non-agglomerate and monodispersed nanoparticles. The synthesized nanoparticles can be stably preserved in the microemulsion system for a long time [12-14].

In this study, the environmental-friendly surfactants sorbitan monooleate (Span 80) and polyoxyethylene (20) sorbitan monostearate (Tween 60) were chosen as mixed surfactants. The water solubilization capacity and the properties of the water molecules solubilized in the interior of the S-T/isooctane system were investigated in order to prepare a transparent, isotropic, and thermodynamically stable $\mathrm{W} / \mathrm{O}$ microemulsion system and then the reverse micelles were used as nanoreactors to synthesize iron nanoparticles, which can be used in environmental contaminants remediation. The properties of the synthetic iron nanoparticles were characterized and analyzed. 


\section{Experiments}

\subsection{Materials and Methods}

Span 80 (chemical grade), Tween 60 (chemical grade), potassium borohydride $\left(\mathrm{KBH}_{4}\right.$, analytical grade), ferrous sulfate heptahydrate $\left(\mathrm{FeSO}_{4} \cdot 7 \mathrm{H}_{2} \mathrm{O}\right.$, analytical grade) were purchased from Fuchen Chemical Reagent Manufactory Tianjin, China. n-Butanol (analytical grade) and isooctane (analytical grade) were supplied by the Sixth Chemical Reagent Manufactory (Tianjin, China) and Kaitong Chemical Reagents Ltd. (Tianjin, China), respectively. All the chemicals were used without further purification. Deionized water was used for aqueous solution and washing in this study.

Morphologies and sizes of the particles were observed with a Philips EM400ST TEM (transmission electron microscopy) (Eindhoven, Netherlands). Crystal structures of the final products were examined with a Rigaku D/max-2500 XRD (X-ray diffraction) (Tokyo, Japan), with $\mathrm{Cu}-\mathrm{K} \alpha$ radiation, voltage was set at $40 \mathrm{kV}$, and current was $100 \mathrm{~mA}(\mathrm{k}=0.1541 \mathrm{~nm})$. The conductivity was measured with a microprocessor conductometer (DDS307, Shanghai Precision and Scientific Instrument Corporation, Shanghai, China). The concentrations of nitrate, nitrite, and ammonium were measured colorimetrically according to the methods 4,500 listed in the 20th edition of the Standard Methods using a UV-Vis spectrophotometer (752N Ultraviolet/Visible Spectrometer, Shanghai Precision and Scientific Instrument Corporation, Shanghai, China) [15].

\subsection{Preparation of Water/Mixed Surfactants/ Isooctane Microemulsion}

Mixed surfactants with different hydrophilic-lipophilic balance (HLB) value were prepared by Span 80 and Tween 60. A given amount of mixed surfactants were mixed with a certain amount of isooctane at a fixed weight ratio, and then dispersed by ultrasound for several minutes. A certain amount of n-butanol used as cosurfactants was added to the above mixture. The conductivity of the system was measured after being dispersed by ultrasound. Deionized water was gradually added into the system by a micro-burette, and the formation of the optically clear microemulsion was determined by conductivity measurement and visual examination.

\subsection{Preparation of Nanoscale Iron}

The iron nanoparticles were formed from the following reaction

$$
\mathrm{Fe}^{2+}+2 \mathrm{BH}_{4}^{-}+6 \mathrm{H}_{2} \mathrm{O} \rightarrow \mathrm{Fe}+2 \mathrm{~B}(\mathrm{OH})_{3}+7 \mathrm{H}_{2} \uparrow
$$

Two water/S-T (Span-Tween mixed surfactants)/ isooctane microemulsions, $\mathrm{A}$ and $\mathrm{B}$, differing only in the types of aqueous phase, were prepared. The microemulsions were both composed of $2.0 \mathrm{~g}$ S-T (Span-Tween mixed surfactants) mixed surfactant, $4.0 \mathrm{~g}$ isooctane, 1.0 $\mathrm{g}$ n-butanol, and $2 \mathrm{~mL}$ aqueous solution. The aqueous phase of microemulsion A was $0.2 \mathrm{~mol} / \mathrm{L} \mathrm{FeSO}_{4}$ solution, whereas the aqueous phase of microemulsion $\mathrm{B}$ was 1.3 $\mathrm{mol} / \mathrm{L} \mathrm{KBH}_{4}$ solutions. Microemulsions A and B were quickly mixed under the protection of argon in a conical flask. The solution turned black after mixing, and was allowed to react for $30 \mathrm{~min}$ with vigorous stirring $(3,000 \mathrm{r} / \mathrm{min})$. At the end of the reaction, the microemulsion system with the well-dispersed iron nanoparticles was transferred into a sealed vessel filled with argon by a double-tipped needle. The resulting black-gray solids were settled by magnet, and the supernatant was decanted. Then the solids were washed with deionized water and finally with a mixture of anhydrous ethanol and acetone (volumetric ratio of ethanol and acetone was 1:1) for six times, respectively. Finally, the resultant blackgray solids were dried under argon atmosphere, and then stored in another sealed vessel filled with argon.

\subsection{Characterization of Particles}

A small amount of microemulsion containing the iron particles were withdrawn from the reaction vessel, and then preserved in a small sealed glass tube. For the TEM investigations, a small drop of microemulsion containing iron nanoparticles was deposited on a copper grid.

The black precipitates formed in the microemulsion were washed with anhydrous ethanol/acetone (volumetric ratio of ethanol and acetone is 1:1) several times and then with deoxygenated and deionized water and finally dried. The entire operation was carried out under an atmosphere of argon. The final dry black solids were analyzed by XRD.

\subsection{Reactivity Assessment}

The performance of the synthetic iron nanoparticles was tested by the reduction of nitrate under anaerobic condition. The synthesized iron nanoparticles react with 80 $\mathrm{mg} / \mathrm{L}$ nitrate solution at initial neutral condition under ambient temperature without $\mathrm{pH}$ control. The concentrations of nitrate were periodically analyzed by ultraviolet (UV) (using Standard Methods) [15].

\section{Results and Discussion}

\subsection{Orthogonal Experiments}

The results of orthogonal experiments indicated that the optimum conditions for the formation of microemulsion system are: hydrophilic-lipophilic balance (HLB) was 14, 
weight ratio of oil and surfactants was 2 , and weight ratio of cosurfactants and surfactants was 0.5 .

\subsection{Phase Behavior of Water/Mixed Surfactants/ Isooctane Microemulsion System}

In order to construct the phase diagram of the water/mixed surfactants/isooctane microemulsion system, the titration method was used. The optimum weight ratio mentioned above was used to prepare the mixed surfactants/n-butanol mixture (SAA-Co). A predetermined amount of isooctane was added to the surfactant mixture. Water was then added into the isooctane/surfactant mixture and changes were observed by visual inspection and conductivity measurement and the information was used to develop a phase diagram. The phase behavior of the water/mixed surfactants/isooctane system is shown in Figure 1. It can be seen in Figure 1 that an ideal W/O region has been observed where weight ratio of n-butanol and mixed surfactants was 1:2, which will be used as the microemulsion system to produce nanoiron particles.

\subsection{Conductivity}

The variation of conductivity with different water content (v) is shown in Figure 2. Two turning points (R1, R2) are found in Figure 2. Almost no change was observed in the initial conductivity of the isooctane/surfactant mixture when $\mathrm{v}$ was smaller than $15 \%$ (R1). This was attributed to the formation of surfactant-bonded water [16] in the system. However, the conductivity increased sharply when $\mathrm{v}$ was bigger than $25 \%$ (R2) upon addition of the water into the isooctane/surfactant mixture for it formed water-in-oil (W/O) microemulsion. In this region, water was present in micelle form and the size and number of water droplets increased with an increased amount of

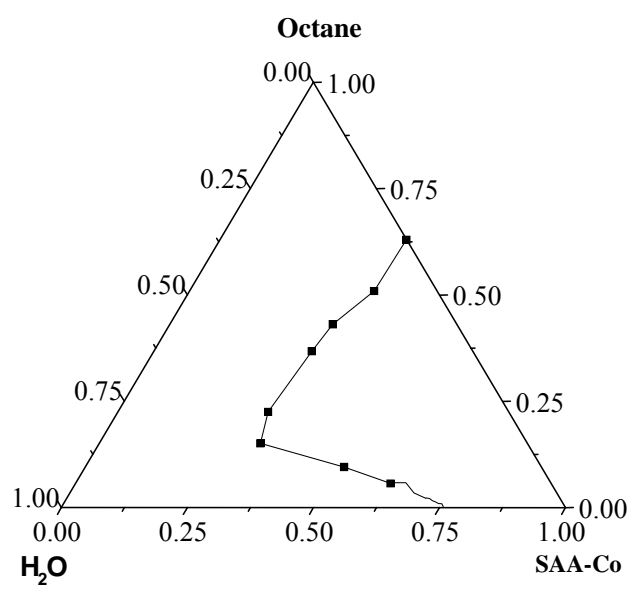

Figure 1. Microemulsion phase diagram of Span80/ Tween60-Isooctane- $\mathrm{H}_{2} \mathrm{O}$ system. water. When the droplet size increased, it was easy to exchange charges between the droplets, and hence the conductivity increased. The conductivity reached the maximum when the water content was $25 \%(\mathrm{wt} \%, \mathrm{R} 2)$. This can be explained by the saturation of droplets and the percolation of charges through the droplet clusters with the minimum resistance. The more water was added to the system when $\mathrm{v}$ was bigger than $25 \%$ (R2), the more phase separation and the conductivity decreased. The optically clear $\mathrm{W} / \mathrm{O}$ microemulsion system that was formed between R1 and R2 can be used as microreactors for preparation of nanoscale iron.

\subsection{Characterization of the Products by Trans- mission Electron Microscopy}

Morphologies of the particles are shown in Figure 3(a)-(f). Figure 3(a) reveals that the synthesized iron nanoparticles dispersed well in the microemulsion system. It can be seen from the TEM images in Figure 3(b) and (c) that the particles were nearly spherical in shape and uniform in size. Figure 3(d) shows clearly the morphology and inner structure of an individual nanoparticle. Apparently, there are two distinct layers as a core-shell -like structure, where the light black and gray shell (thickness of about $10 \mathrm{~nm}$ ) is probably due to S-T mixture while the core with the thickness of about $80^{-90} \mathrm{~nm}$ is attributed to $\mathrm{Fe}^{0}$.

Comparing Figure 3(e) with (f), the iron nanoparticles were not so well-dispersed after breakdown of the microemulsion by washing. The individual small iron nanoparticles aggregate to significantly larger nanoparticles after being separated from the "water pools", and had an irregularly spherical shape. This is because that the S-T mixture shell was destroyed in the washing procedure, and thus the mobility of particles increased.



Figure 2. Variation of conductivity with amount of water in microemulsion. 


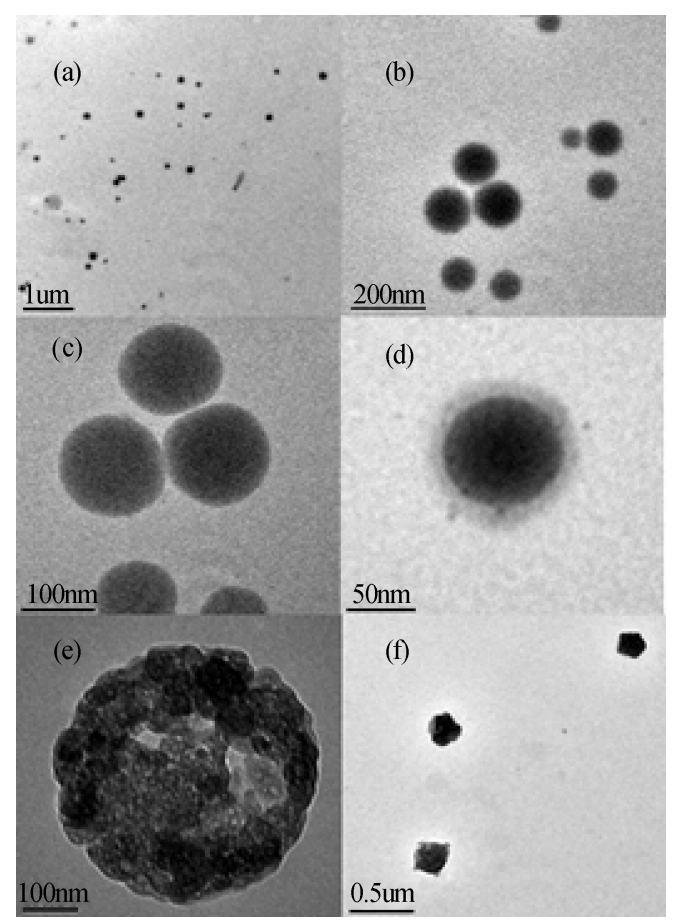

Figure 3. TEM images of Fe ultrafine particles (a), (b), (c), (d) Iron particles before demulsification; (e), (f) iron particles after demulsification.

\subsection{X-Ray Diffraction Results}

Figure 4 is the X-ray diffraction pattern of nanoiron and shows that crystal iron particles existed in the product. The XRD pattern shows characteristic broad peaks, which is consistent with the $\alpha$-Fe standard pattern in the range of $20^{\circ}-80^{\circ}$; and therefore, it is concluded that the nanoiron synthesized in this work was mainly composed of $\alpha$-Fe.

\subsection{Results of Reactivity Assessments}

The reaction between nanoiron and nitrate was used to evaluate the reactivity of the synthesized iron nanoparticles. Freshly synthesized iron nanoparticles $(0.25 \mathrm{~g})$ were charged into $125 \mathrm{~mL}$ solution containing $80 \mathrm{mg} / \mathrm{L}$ nitrate which was prepared in the laboratory. The black iron nanoparticles were quickly solubilized (the system turned reddish-brown when there is oxygen in it), which indicated that the freshly synthesized iron nanoparticles reacted instantly with oxidative matters in the solution. Freshly synthesized iron nanoparticles reacted with nitrate much faster than reduced $\mathrm{Fe}^{0}$ powder under the same condition. On neutral condition, commercially available $\mathrm{Fe}^{0}$ powder did not react with nitrate in 120 min, whereas complete denitrification of nitrate was achieved by freshly synthesized nanoiron in $30 \mathrm{~min}$ (Figure 5). A smaller particle size and much higher spe- cific surface area of nanosized zero-valent iron as compared with the reduced $\mathrm{Fe}^{0}$ powder should account for the high reduction rate for nitrate by nanoscale iron used in this study. Compared with microscale particles, the superficial atom ratio of nanoscale particles dramatically increased from $1 \%-2 \%$ to more than $50 \%$, resulting in greatly increased chemical reactivity, high specific surface area, and superior surface reactivity. Thus, nanoscale iron contacted and reacted more easily with contaminants than general $\mathrm{Fe}^{0}$ powder.

\subsection{Proposed Reaction Pathways}

Figure 6 reveals that ammonia was the predominant endproduct of the reaction between nanoscale iron and nitrate. Only small amounts of nitrite were detected in the reaction process, and rapid transformation to ammonia was observed subsequently. It can be seen from the chemical mass balance curve that total nitrogen (sum of nitrate, nitrite, and ammonia) displayed a minor dip at the initial stage (from 0 to $10 \mathrm{~min}$ ) of nitrate reduction, indicating that the reaction mechanisms were complex.

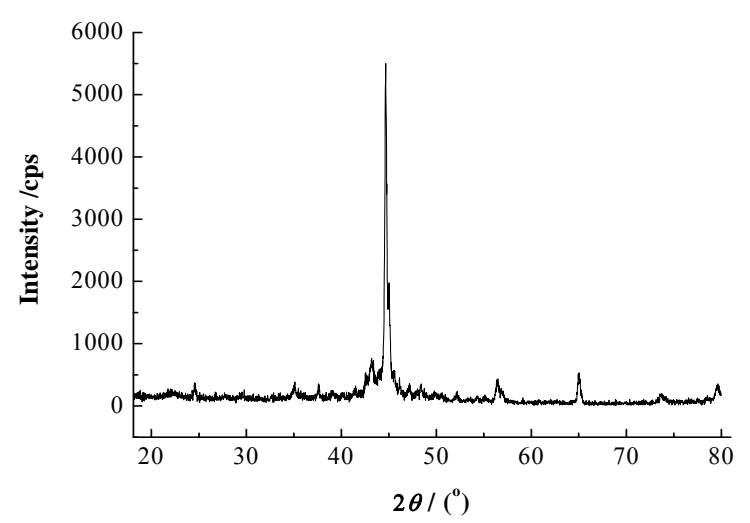

Figure 4. X-ray diffraction (XRD) pattern of Fe ultrafine particles.

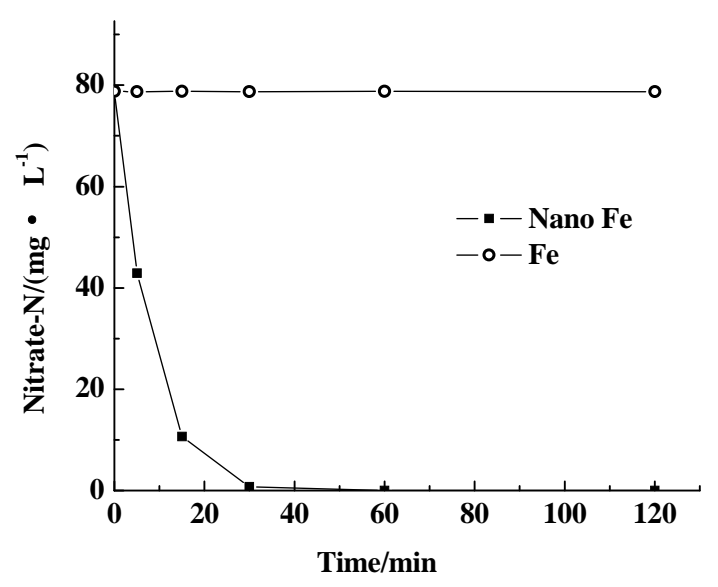

Figure 5. Reactions of nitrate with commercial Fe powders (Fe), nanoscale Fe particles (nano Fe) at pH 7. 
Therefore, two possible reaction pathways were proposed for nitrate reduction by nanoscale zero-valent iron as shown in Figure 7: 1) directly reduce to ammonia and 2) first adsorb to the nanoiron surface and then reduce to ammonia. The adsorption of nitrate onto nanoiron surface resulted in total nitrogen (in aqueous solution) decreasing at first. However, the amount of nitrate which adsorbed to the nanoiron surface was rapidly reduced to ammonia, and then desorbed into the solution from iron surface again, so total nitrogen in the solution increased nearly to the initial level at the end of the reaction. So, the reaction pathway of nitrate reduction by nanorion is the second one.

\subsection{Reaction Kinetics of Nitrate}

To study the kinetics of denitrification by nanoscale iron, the effect of nitrate concentrations on the nitrate removal rate was observed. Four different initial nitrate concentrations $(30,50,80,120 \mathrm{mg} / \mathrm{l})$ were tried and the changes in nitrate concentrations with reaction time are given in Figure 8. For avoiding the effect of changes of $\mathrm{Fe}^{0}$ concentration by corrosion on the kinetics during the reaction, stoichiometric excess of $\mathrm{Fe}^{0}$ particles compared with nitrate applied were added.



Figure 6. Mass balance of nitrate reduction with nanoscale Fe.

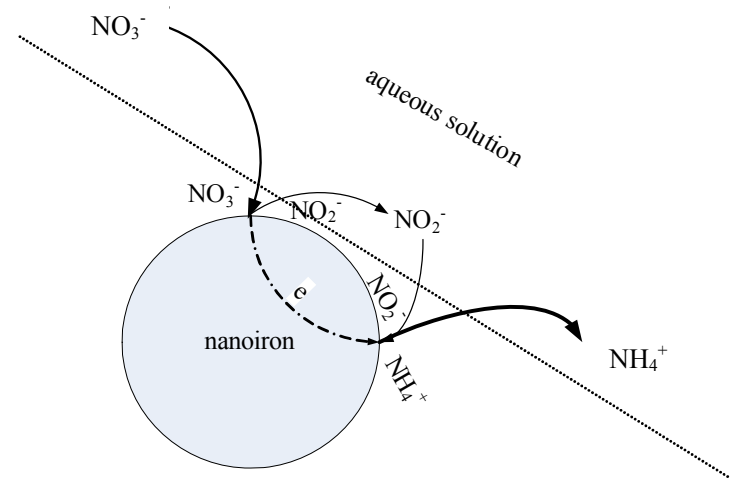

Figure 7. Pathways of nitrate reduction by nanoscale Fe.



Figure 8. Reaction of nanoscale iron particles with different initial $\mathrm{NO}_{3}{ }^{-} \mathrm{N}$ concentration solution.

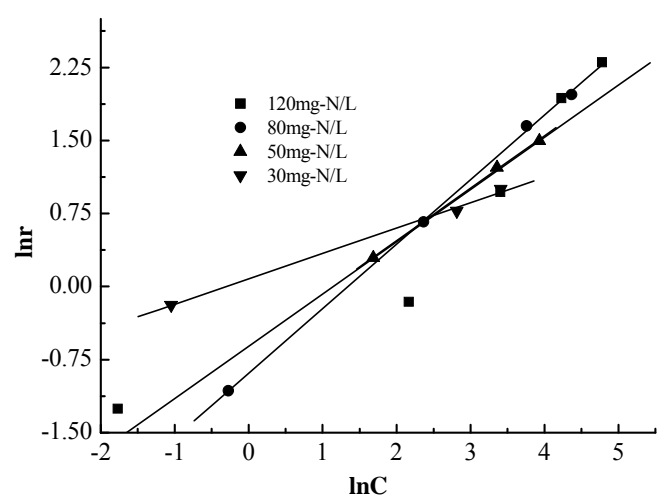

Figure 9. Plot of $\ln -\mathrm{lnC}$ under different initial $\mathrm{NO}_{3}{ }^{-} \mathrm{N}$ concentration.

Table 1. Reaction rate equation for reduction of nitrate having different initial concentrations by nanoscale ZVI.

\begin{tabular}{cccc}
\hline $\begin{array}{c}\text { Initial conc. } \\
\text { of nitrate } \\
\text { mg-N/L }\end{array}$ & $\begin{array}{c}\text { Reaction } \\
\text { order } \\
\mathrm{n}\end{array}$ & $\begin{array}{c}\mathrm{K}_{\text {obs }} \\
(\mathrm{mg}-\mathrm{N} / \mathrm{L})^{1-\mathrm{n}} \cdot \mathrm{min}^{-1}\end{array}$ & $\mathrm{R}$ \\
\hline 30 & 0.26 & 1.08 & 0.9986 \\
50 & 0.54 & 0.55 & 0.9995 \\
80 & 0.66 & 0.41 & 0.9997 \\
120 & 0.54 & 0.54 & 0.9496 \\
\hline
\end{tabular}

Excluding the effect of iron concentration, the reaction can be described as follows:

$$
\begin{gathered}
r=\frac{-d C}{d t}=k_{o b s} C^{n} \\
\ln r=\ln \left(\frac{-d C}{d t}\right)=\ln \left(k_{o b s} \cdot C^{n}\right)=\ln k_{o b s}+n \ln C
\end{gathered}
$$

where $\mathrm{r}$ is reaction rate, $k_{o b s}$ is the observed reaction rate constant, $\mathrm{n}$ is reaction orders. Plot the $\ln k_{o b s}$ values vs $\operatorname{lnr}$. The good fit of the linear model to data was observed from Figure 9. And, the slopes of these lines result in apparent reaction orders. The values of $n$ were observed in Table 1. Furthermore, the same result was also observed in other studies of chemical reduction of nitrate by nanosized iron [16]. 


\section{Acknowledgment}

This work was supported by Doctoral Fund for Young Scholar of Ministry of Education of China (No. 20070055053) and the Tianjin Natural Science Foundation of China (Grant No. 07JCZDJC01800).

\section{References}

[1] C. P. Zhang, W. Deng, Z. C. Hu, Y. P. Luo, X. Gao, and L. $\mathrm{Hu}$, "Preparation of ultrafine $\mathrm{Fe}$ particles by microemulsion method," Chinese Journal of Applied Chemistry, Vol. 17, No. 3, pp. 248-251, 2000. (in Chinese)

[2] Y. H. Deng, L. Wang, W. L. Yang, S. K. Fu, and E. Abdelhamid, "Synthesis of 30-100 nm nanoscale magnetic polymer particles in inverse microemulsion," Chemical Journal of Chinese Universities, Vol. 24, No. 5, pp. 920-923, 2003. (in Chinese)

[3] S. X. Zhang, C. P. Zhang, H. Gou, Y. P. Luo, J. Liu, and D. J. Shen, "Preparation of gelatin-nickel-iron composite nanoparticles with gel-microemulsion chemical tailoring (II) effect of relevant parameters on the composition and size of particles and magnetic properties," Chinese Journal of Rare Metals, Vol. 27 No. 4, pp. 429-433, 2003. (in Chinese)

[4] W. X. Zhang, "Nanoscale iron particles for environmental remediation: An overview," Journal of Nanoparticle Research, Vol. 5, No. 3-4, pp. 323-332, 2003.

[5] F. Li, C. Vipulanandan, and K. K. Mohanty, "Microemulsion and solution approaches to nanoparticle iron production for degradation of trichloroethylene," Colloids and Surfaces A: Physicochemical and Engineering Aspects, Vol. 223, No. 1-3, pp. 103-112, 2003.

[6] C. B. Wang and W. X. Zhang, "Synthesizing nanoscale iron particles for rapid and complete dechlorination of TCE and PCBs," Environmental Science \& Technology, Vol. 31, No. 7, pp. 2154-2156, 1997.

[7] H. L. Lien and W. X. Zhang, "Nanoscale iron particles for complete reduction of chlorinated ethenes," Colloids and
Surface A: Physicochemical and Engineering Aspects, Vol. 191, No. 1-2, pp. 97-105, 2001.

[8] S. R. Kanel, J. M. Greneche., and H. Choi, "Arsenic (V) removal from groundwater using nanoscale zero-valent iron as a colloidal reactive barrier material," Environmental Science \& Technology, Vol. 40, No. 6, pp. 2045-2050, 2006.

[9] S. M. Ponder, J. Darab, and T. E. Mallouk, "Remediation of $\mathrm{Cr}(\mathrm{VI})$ and $\mathrm{Pb}(\mathrm{II})$ aqueous solutions using supported nanoscale zerovalent iron," Environmental Science \& Technology, Vol. 34, No. 12, pp. 2564-2569, 2000.

[10] L. W. Chen, L. H. Gan, and T. Y. Yue, "Studies on the preparation of aluminium oxide (hydrous) ultrafine particles by the method of microemulsion reaction," Chemical Journal of Chinese Universities, Vol. 16, No. 1, pp. 13-16, 1995. (in Chinese)

[11] J. Z. Andrey and Y. Y. Jackie, "Reverse microemulsion synthesis of nanostructured complex oxides for catalytic combustion," Nature, Vol. 403, No. 6, pp. 65-68, 2000.

[12] C. L. Chiang, M. B. Hsu, and L. B. Lai, "Controlled of nucleation and growth of gold nanoparticles in AOT/Span 80/ isooctane mixed reverse micelles," Journal of Solid State Chemistry, Vol. 177, No. 11, pp. 3891-3895, 2004.

[13] C. Y. Wang, W. Q. Jiang, Y. Zhou, Y. N. Wang, and Z. Y. Chen, "Synthesis of $\alpha$-Fe ultrafine particles in a saturated salt solution/isopropanol/PVP microemulsion and their structural characterization," Materials Research Bulletin, Vol. 35, No. 1, pp. 53-58, 2000.

[14] H. L. Lien and W. X. Zhang, "Nanoscale iron particles for complete reduction of chlorinated ethenes," Colloids and Surfaces A: Physicochemical and Engineering Aspects, Vol. 191, No. 1, pp. 97-105, 2001.

[15] Q. Li, Y. Li, W. H. Li, S. F. Weng, J. G. Wu, and G. X. $\mathrm{Xu}$, "FT-IR study of water structure in water/NaDEHP/ n-heptane microemulsion system," Journal of Peking University (Natural Science), Vol. 33, No. 4, pp. 410-415, 1997. (in Chinese)

[16] G. C. Yang and H. L. Lee, "Chemical reduction of nitrate by nanosized iron: kinetics and pathways [J]," Water Research, Vol. 39, No. 5, pp. 884-894, 2005. 